\section{Proteomic analysis and sperm physiopathology: the two-dimensional difference in gel electrophoresis approach}

Two-dimensional (2D) gel electrophoresis coupled with protein identification using mass spectrometry (MS) is a procedure extensively used to obtain a catalog of several thousand proteins in a single gel. These evaluations provide information that complements and extends the results retrieved from messenger RNA (mRNA) profiling using microarray technologies (1). Basically, the first step on the $2 \mathrm{D}$ gel electrophoresis analysis is based on protein separation according to its isoelectric point (pI). The $\mathrm{pI}$ is the $\mathrm{pH}$ at which the protein carries no net charge; consequently, when proteins are subjected to a $\mathrm{pH}$-gradient electrophoresis, those with a net negative charge migrate to the anode, and those with a positive charge migrate to the cathode, until the $\mathrm{pH}$ of the gradient is equal to the protein $\mathrm{pI}$ and the overall net charge is zero.

The second (orthogonal) dimension is based on differences in protein electrophoretic mobility due to its size. It uses the traditional technique of polyacrylamide gel electrophoresis (PAGE) containing sodium dodecyl sulphate (SDS) as a protein denaturing agent (SDS-PAGE).

The 2D gel proteomic procedure has been successfully applied in human and animal models to characterize the protein repertoire of numerous biologic systems. In the reproductive biology field, several proteomic techniques have been applied in the last decade to obtain a protein catalog of the male gamete (2).

Classic 2D-gel based proteomic techniques allow the detection of differences in the protein signal that reflect changes in the expression levels between some experimental or clinical conditions. Moreover, it may reveal modifications in the molecular weight and/or pI of a specific protein that have resulted from processing and charge-altering posttranslational modifications (such as glycosylation, phosphorylation, sulfation, and acetylation/deactylation) of biologic significance. However, reproducibility of protein profiles requires additional computerized work to justify 2D gel images and allow their comparison, reducing the speed and accuracy of quantitation.

To overcome some of these limitations, the $2 \mathrm{D}$ difference in gel electrophoresis (2D-DIGE) is a variant of 2D gel electrophoresis procedure that provides an approach to linking samples across different gels and to comparing protein expression accurately in two defined conditions. The concept of 2D-DIGE was originally developed in the late 1990s by Minden et al. (3); since then, it has been extensively applied in numerous studies. Basically, two protein pools are labeled with cyanine (Cy3 and Cy5) fluorescent dyes in a controlled amount to label one protein molecule of the cell extract or fluid mixture not more than once; in addition, a control pool is prepared labeling proteins from both samples under study with the $\mathrm{Cy} 2$ dye. These compounds are N-hydroxy succinimidyl ester derivatives specifically designed to react with the $\varepsilon$-amino group of lysine in the protein. The labeled proteins are mixed and separated in the same 2D gel; once separation is complete, the gel is subjected to analysis by obtaining images of protein patterns after fluorescence dye excitation (CyDye technique) and signal quantitation of each protein spot. Protein identification is achieved after spot picking of selected components to show a differential expression (evidenced by changes in signal intensity in each condition), followed by protein digestion and MS analysis. Validation studies using quantitative Western immunoblotting are run to confirm differences in expression levels of specific components found with peptide analysis.

The 2D-DIGE methodology, a step forward for highthroughput 2D gel analysis, is very advantageous when compared with a conventional 2D gel separation. One-half of the gels are subjected to analysis because two sample pools are separated in the same gel. The procedure shows several additional advantages:

1. Improved procedure time. The CyDye detection technique is less time consuming than the silver and Coomassie brilliant blue (CBB) staining techniques in which gels need to be fixed and stained/destained.

2. Improved reproducibility. Two protein pools are separated in the same gel, and proteins from both pools are subjected to the same conditions, anticipating similar protein behavior with same location in the 2D gel.

3. Improved sensitivity and wider dynamic range. The CyDye technique is a very sensitive method for protein detection (low nanogram) and covers a wider dynamic range (four orders of magnitude) than silver and CBB conventional staining procedures.

4. Accurate repetitive measurements. Strategies can be applied to perform repetitive measurements and multivariable analyses in one experiment, which positively impacts in the statistical data analysis.

5. Improved comparison. Protein expression pattern analyses of replicates in different gels are simplified because internal controls (Cy2 sample pool) are part of the experimental design and different experimental/clinical conditions are included in the same run.

6. Rapid quantitation. Fast quantitation of protein profiles is achieved in an accurate fashion based on the fluorescence intensity of the dyes coupled to the proteins. Reports have been already published on the use of this technology in the evaluation of sperm physiology as well as in specific pathological situations (4).

Despite the advantages of the 2D-DIGE procedure, some technical limitations should be taken into account when using this proteomic technology because the fluorescence dyes used for identification and quantitation modify the protein patterns obtained with the 2D-DIGE system compared with those obtained when conventional approaches. Specifically, proteins without lysine are not labeled, so a high abundance protein spot in a conventional gel system may be absent in the 2D-DIGE protein profile. In fact, only a small percentage of the protein is labeled, and most of the protein molecules cannot be visualized in the Cy3 or Cy5 images.

Moreover, protein labeling may have different efficiencies depending on lysine content (proteins with a high percentage of lysine residues may be more efficiently labeled 
than proteins containing little lysine). In this regard, addition of the dye slightly changes protein migration because it adds approximately $0.5 \mathrm{kDa}$ to the total molecular protein mass, affecting more small than large proteins. Finally, highly acidic, basic, or hydrophobic proteins as well as small (less than $15 \mathrm{kd}$ ) proteins or peptides and low-abundant proteins are difficult to identify with 2D-DIGE because of technical limitations. The 2D-DIGE procedure can be performed in conjunction with other techniques, such as MS-based approaches using differentially labeled stable isotopes, to improve and expand protein identification in complex protein mixtures.

Hamada et al. (5), in this issue of Fertility and Sterility, describe the use of the 2D-DIGE proteomic analysis in a protein global evaluation of spermatozoa from human semen samples with high and low levels of reactive oxygen species (ROS + ve and ROS-ve, respectively) and the relationship between changes in the protein profile and ROS levels. The study involved the evaluation of sperm proteins from semen samples of 20 donors and 32 infertile men, which were pooled, divided into ROS + ve and ROS - ve groups $(<20$ relative light units $/$ sec/million spermatozoa), frozen, and later processed using 2D-DIGE and MS analysis. From a total of 1,343 protein spots detected in gel 1 (ROS-ve) and 1,265 spots in gel 2 (ROS+ve), 31 spots were detected as differentially expressed (1.5 fold). Of those, 25 spots were statistically significantly increased and six spots decreased in the ROS-ve pool sample compared to the ROS+ve pool sample. Of the 25 spots, the investigators reported the identification of lactotransferrin 2 (NCB 312433998) and peroxiredoxin-1 (NCB 4505591). They discuss the relevance of the findings in connection with previous reports describing ROS metabolism and sperm function, and they propose future validation studies of the identified proteins (5).

After successful completion of the Human Genome Project, the Human Proteome Project (HPP) has been officially launched with the aim to map the entire human protein cata- log. This exciting new challenge for the biomedical field will undoubtedly provide relevant information to better understand the male gamete at the molecular level and, hopefully, will contribute to the management of male infertility.

Mónica H. Vazquez-Levin, Ph.D.

National Research Council of Argentina (CONICET), Instituto de Biología y Medicina Experimental, Buenos Aires, Argentina

http://dx.doi.org/10.1016/j.fertnstert.2012.12.051

You can discuss this article with its authors and with other ASRM members at http://fertstertforum.com/vazquezlevinm-proteomicanalysis-sperm-physiopathology-2d-dige/

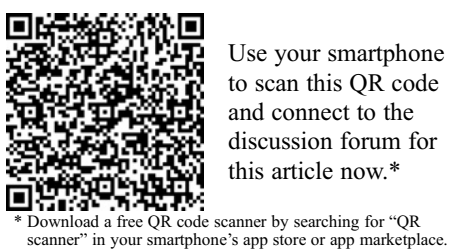

\section{REFERENCES}

1. Weiss W, Görg A. High-resolution two-dimensional electrophoresis. Methods Mol Biol 2009;564:13-32.

2. de Mateo S, Estanyol JM, Oliva R. Methods for the analysis of the sperm proteome. Methods Mol Biol 2013;927:411-22.

3. Unlü M, Morgan ME, Minden JS. Difference gel electrophoresis: a single gel method for detecting changes in protein extracts. Electrophoresis 1997;18: 2071-7

4. Liao TT, Xiang Z, Zhu WB, Fan LQ. Proteome analysis of round-headed and normal spermatozoa by 2-D fluorescence difference gel electrophoresis and mass spectrometry. Asian J Androl 2009;11:683-93.

5. Hamada A, Sharma R, Du Plessis S, Willard B, Yadav SP, Sabanegh E, Agarwal A. Two-dimensional differential in-gel electrophoresis-based proteomics of male gametes in relation to oxidative stress. Fertil Steril 2013;99: 1216-26. 